\title{
BEHAVIORAL AND EMOTIONAL REACTIONS OF THE RUSSIAN POPULATION TO THE BEGINNING OF THE COVID-19 PANDEMIC: AN ON-LINE SURVEY RESULTS
}

\author{
Mikhail Yu. Sorokin ${ }^{1}$, Evgeny D. Kasyanov ${ }^{1}$, Grigory V. Rukavishnikov ${ }^{1}$, Olga V. Makarevich ${ }^{1}$, \\ Nikolay N. Neznanov ${ }^{1,2}$, Natalia B. Lutova ${ }^{1}$, Galina E. Mazo ${ }^{1}$ \\ ${ }^{I}$ V.M. Bekhterev National Medical Research Center for Psychiatry and Neurology, Saint-Petersburg, Russia \\ ${ }^{2}$ I.P. Pavlov First Saint-Petersburg State Medical University, Saint-Petersburg, Russia
}

received: 18.4.2021;

revised: 17.7.2021;

accepted: 29.7 .2021

\section{SUMMARY}

Background: The study aimed to identify the patterns of adaptive and behavioral strategies in different population groups, also to evaluate their association with the infection prevention strategies and the distress during the COVID-19 pandemic.

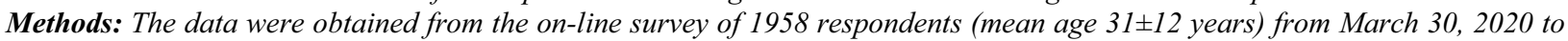
April 5, 2020. 578 respondents reported a history of affective disorders; 884 respondents - a history of somatic disease. The level of anxiety distress was evaluated with the Psychological Stress Measure (PSM-25). The analysis of variance was used for statistics. $p<0.05$ was considered significant. The effect sizes (ES) were evaluated according to Cohen's $d$ and Cramer's V criteria.

Results: The average PSM-25 score corresponded to moderate stress intensity. An increased level of psychological stress was associated with the young age of the respondents, the history of affective disorders and somatic diseases, the compliance with selfisolation, the practice of social distancing, and the use of sanitizer. Concerns about the availability of protective equipment were specifically associated with the self-isolation compliance (ES=0.1); the combination of concerns about the contagiousness of the virus $(E S=0.12)$ and the inaccessibility of daily medications $(E S=0.11)$ - with the principles of social distance. Moreover, the concerns about the lack of specific treatment, the danger to one's own life, the contagiousness of the virus, and the lack of protective equipment were associated with the protective behavior resulting in increased hand hygiene. The history of affective disorders was rarely associated with wearing masks and gloves, but more often - with the use of self-isolation strategies.

Conclusions: The psychological reactions of the population during the COVID-19 pandemic are specifically associated with adaptive behavior in the process of anti-epidemic measures. Respondents with affective disorders experienced specific patterns of anxiety about coronavirus infection in combination with high rates of psychological stress.

Key words: COVID-19 - anxiety - distress - behavior patterns - initial pandemic stages

Abbreviations: COVID-19: Coronavirus disease of 2019; PSM-25: Psychological Stress Measure

\section{INTRODUCTION}

The first cases of the novel coronavirus infection COVID-19 (COrona VIrus Disease 2019) were identified in December 2019. In several months the disease rapidly spread around the world, causing a pandemic. Until now, the number of infection cases and deaths continues to grow worldwide. In the Russian Federation, the first individuals were diagnosed with COVID19 on January 31, 2020, and in December more than 3,000,000 Russians had confirmed diagnoses (Center for Systems Science and Engineering (CSSE) at Johns Hopkins University 2020). Since March 2020, many governments have introduced quarantine measures to limit the spread of the virus and minimize the burden on health services (Brooks et al. 2020). According to the available preliminary data, the elderly with concomitant somatic pathology were more prone to infection and its severe course (Wang et al. 2020).

In the context of the necessary epidemiological limitations, the community of mental health professionals is trying to identify the most significant factors of dysfunc- tional behavior in the population. This is likely to help mobilizing health resources to provide adequate and personalized care to the individuals (Duan \&Zhu 2020).

Any major epidemic outbreak has negative consequences for both individuals and a whole society. One of the many negative consequences of the COVID-19 pandemic is the "second epidemic" of negative psychological effects (Huang \& Zhao 2020). It can contribute to the further deterioration of the epidemiological process and requires early intervention at the initial stages (Li et al. 2020).

Evaluating psychological and behavioral responses during the quarantine early stages can be the key to corrective and preventive strategies. However, despite the high interest in the evaluation of stress levels in the population during a pandemic, there is few data about the initial stages of quarantine measures. The data from Spanish research of 1003 respondents interviewed at the initial stage of quarantine showed a high level of affective reactions in society (Ozamiz-Etxebarria et al. 2020). However, these studies focused only on assessing stress levels and did not address the associated behavioral patterns. 
In psychology of the health there are 2 patterns considering: a) the rational causes of human behavior; b) irrational behavior which is difficult to predict (Rasskazova \& Ivanova 2015). In the context of COVID-19 pandemic, there is already evidence of pragmatic reasons for the population's compliance with anti-epidemic measures (Moore et al. 2020, Singh \& Adhikari 2020).

However, due to the exceptional social importance of adherence with anti-epidemic measures by the majority of the population, it is necessary to understand all potential factors of adherence / non-adherence individual sub-cohorts.

Hypothesis: Population behavioral patterns in antiepidemic measures are associated with clinical, psychological and demographic factors.

Objective: To identify the characteristics of emotional and behavioral reactions associated with the onset of the COVID-19 pandemic in different population groups.

\section{MATERIALS AND METHODS}

\section{Study design and setting}

The data were obtained from the online survey performed from March 30 to April 5, 2020 (first week of quarantine measures in Russia). The respondents were proposed to complete the self-reported Questionnaire via the Google Forms online platform, which on average took about 15 minutes. The Questionnaire was distributed through social networks and websites of public organizations and thematic communities (see Acknowledgements).

Inclusion criteria were: age $\geq 18$, ability to read and understand text in Russian; submitted consent to personal data processing. Exclusion criteria: any blank sections in the Questionnaire forms.

The Questionnaire included social and demographic data about participants, information about the history of affective disorders (major depressive disorder, bipolar affective disorder, generalized anxiety disorder, cyclothymia, dysthymia) and somatic pathology based on respondent's self-reports. The participants also choose from several Questionnaire items describing different types of concerns associated with COVID-19 (contagiousness of the virus; risk of isolation; absence of specific treatment for COVID-19; fear for self-life; risk to the lives and health of relatives; possible financial difficulties; severe social consequences; lack of safety equipment for sale; possible lack of medication for daily intake; impossibility of traditional way of life) and behavioral patterns of infection prevention (wearing a mask or respirator; use of antiseptics; hand washing; social distance; self-isolation). The Psychological Stress Measure (PSM-25) was used for quantitative assessment of psychological stress.

The study was approved by local IRB, all procedures were performed in accordance with the WMA Declara- tion of Helsinki. The written online consent was taken and participants were asked to fill the online questionnaire only if they provide consent at the beginning of questionnaire.

\section{Statistical analysis}

Statistical data processing was performed using the SPSS-16 software package (SPSS Inc.; USA). The descriptive statistics were used. Distribution normality test performed using the skewness and kurtosis calculation. Dispersion analysis for data with nominal scales analyzed using Pearson's $\chi^{2}$ criterion, the data for ordinal scales analyzed using Kruskal-Wallis $\mathrm{H}$ test and Mann-Whitney U-criterion, and the data for interval scales analyzed using ANOVA. The results are given with an indication of average $\mathrm{M}$ (S.D.), as well as median and interquartile range for nominal scales for the purpose of data presentation uniformity. Effect sizes obtained using Cohen's d and Cramer's V measures were calculated for groups, the differences between which had the significance level $p \leq 0.05$. Estimation of the effect size was made according to generally accepted criteria: weak 0.10-0.29, moderate 0.3-0.49, strong $\geq 0.50$. The interpretation of the effect size was corrected for the number of freedom degrees and the threshold values for a weak/medium/strong effect when comparing the nominal attributes with more than two gradations.

\section{RESULTS}

\section{Characteristics of study sample}

The final sample included 2117 records completed during the first week of recommended self-isolation in Russia (from March 30 to April 5). The data of 160 respondents were excluded due to age criteria. Thus, the data of 1957 participants from federal cities (SaintPetersburg - 21.1\%, Moscow - 16.8\%), 57.6\% respondents of all other Russian federal districts and 4.5\% responses from other countries were included in the study sample. The majority of respondents were women (1649 persons, $84.3 \%$ ). The average age of the respondents was 31(12) years (stratification by age group see Table 1). 31 participants $(1.6 \%)$ had incomplete secondary education, 98 - secondary education (5\%), professional - $164(8.4 \%)$, incomplete higher education - $501(25.6 \%)$, higher - 1082 (55.3\%), academic degree - $81(4.1 \%)$. The following occupation variants of study respondents were identified at the time of the survey: students - 271 people (13.85\%), unemployed - $435(22.2 \%)$, private sector employee $462(23.6 \%)$, and public sector employee - 631 $(32.2 \%)$, businessman - 158 respondents $(8.1 \%)$. The history of concomitant somatic pathology was reported by $45.2 \%$ respondents, affective disorders - by $29.5 \%$. 
Table 1. The prevalence of psychological distress in the evaluated subgroups of respondents

\begin{tabular}{|c|c|c|c|}
\hline \multicolumn{2}{|c|}{$\begin{array}{l}\text { Subgroups of respondents } \\
\text { (the number of participants) }\end{array}$} & \multirow{2}{*}{$\begin{array}{c}\text { PSM-25 } \\
\text { (M(S.D.) / Cohen's d) } \\
121.2(32.4) / 0.49\end{array}$} & \multirow{2}{*}{$\begin{array}{c}\text { Significance level of group differences } \\
\mathrm{p} \leq 0.003\end{array}$} \\
\hline Age, years & $18-20(n=310)$ & & \\
\hline & $21-30(n=859)$ & $112.3(32.2) / 0.22$ & $\mathrm{p} \leq 0.003$ \\
\hline & $31-40(n=363)$ & $96.7(33.8) /-0.24$ & $\mathrm{p} \leq 0.02$ \\
\hline & $41-50(n=231)$ & $87.1(29.3) /-0.56$ & $\mathrm{p} \leq 0.02$ (except groups $51-78$ years) \\
\hline & $51-60(n=136)$ & $85.0(30.1) /-0.62$ & $\mathrm{p} \leq 0.02$ (except groups $41-50,61-78$ years) \\
\hline & $61-78(n=58)$ & $76.0(24.3) /-0.97$ & $\mathrm{p} \leq 0.02$ (except group $41-60$ years) \\
\hline \multirow[t]{2}{*}{ Affective disorder } & Yes $(\mathrm{n}=578)$ & $127.9(29.1) / 0,72$ & $\mathrm{p}=0.000$ \\
\hline & No $(\mathrm{n}=1379)$ & $95.2(31.7) /-0.29$ & - \\
\hline \multirow[t]{2}{*}{ Somatic pathology } & Yes $(n=884)$ & $108.0(34.7) / 0.09$ & $\mathrm{p}=0.000$ \\
\hline & No $(n=1073)$ & $102.3(33.9) /-0.08$ & - \\
\hline
\end{tabular}

The age distribution of the study participants who reported the history of affective disorders (by age subgroups: $22.6 \%, 55.5 \%, 14.4 \%, 1.9 \%, 1.6 \%, 0 \%)$ differed significantly from respondents without mental disorders history $(11.3 \%, 39.0 \%, 20.3 \%, 16.0 \%, 9.2 \%$, $4.2 \%$, respectively, Pearson's $\chi^{2}=220.3, p=0.000$; Cramer's $\mathrm{V}=0.34$ ). The subgroup of persons with somatic pathology on the contrary was predominantly represented by more mature respondents (by age groups $13.9 \%, 42.2 \%, 15.5 \%, 13.8 \%, 10.3 \%, 4.3 \%$ and $17.4 \%$, $45.3 \%, 21.1 \%, 10.2 \%, 4.2 \%, 1.9 \%$ respectively; Pearson's $\chi^{2}=54.0, \mathrm{p}=0.000$; Cramer's $\mathrm{V}=0.17$ ). The study participants distribution by health groups satisfied to the principle of randomness: healthy $-37.6 \%$, with affective disorders $-17.2 \%$, with somatic burden $-32.8 \%$, with comorbid somatic and mental pathology $-12.4 \% ; \chi^{2}$ Pearson $=3.7, \mathrm{p}=0.06$ ).

\section{Psychological and behavioural reactions of respondents to the pandemic}

The average score on the PSM-25 scale reached 104.9 (34.4) points in the whole sample, which corresponded to the average stress intensity. The results of stress assessment in the selected subgroups of respondents are presented in Table 1.

Associations of certain types of coronavirus-related anxiety concerns with the preventive behavior were both specific and non-specific (Table 2). Thus, concerns about the threat to the self-life and relative's health during the pandemic was not typical to one certain behavior strategie, but accompanied a wide list of the most common protective measures in the sample (hand washing (Pearson's $\chi^{2}=51.5, p=0.000$; Cramer's $\mathrm{V}=0.16$ ), adherence to the principles of self-isolation (Pearson's $\chi^{2}=25.2, p=0.000$; Cramer's V=0.11) and physical distancing (Pearson's $\chi^{2}=38.6, \mathrm{p}=0.000$; Cramer's V $=0.14$ ), the use of sanitizers (Pearson's $\chi^{2}=69.4, p=0.000$; Cramer's V $=0.19$ ).

A number of other concerns about the pandemic have been associated with increased prevalence of some protective measures, and these associations have been more specific. For example, the proportion of respon- dents on self-isolation was higher if they had concerns about the lack of commercially available protective remedies (Pearson's $\chi^{2}=20.0, p=0.000$; Cramer's $V=0.10$ ). Adherence to the principles of social distancing in everyday life was characteristic for the combination of concerns about virus transmissibility (Pearson's $\chi^{2}=29.5, \mathrm{p}=0.000$; Cramer's V=0.12) and the lack of medication for daily use (Pearson's $\chi^{2}=22.7, p=0.000$; Cramer's V=0.11).

The prevalence of protective behaviours patterns associated with hand hygiene (washing, sanitizer use) was positively associated with a wide range of concerns (lack of specific treatment for COVID-19 (Pearson's $\chi^{2}=35.4, p=0.000$, Cramer's V=0.13; Pearson's $\chi^{2}=29.7$, $\mathrm{p}=0.000$, Cramer's V $=0$ ). 12 ), virus transmissibility (Pearson's $\chi^{2}=30.1, \mathrm{p}=0.000$, Cramer's V $=0.12$; Pearson's $\chi^{2}=33.0, p=0.000$, Cramer's $\mathrm{V}=0.13$ ), threat to the selflife (Pearson's $\chi^{2}=24.6, p=0.000$, Cramer's $V=0.13$ ). 11; Pearson's $\chi^{2}=30.1, p=0.000$, Cramer's V $=0.12$ ) and lack of commercially available protection remedies (Pearson's $\chi^{2}=23.0, \mathrm{p}=0.000$, Cramer's V $=0.11$; Pearson's $\chi^{2}=51.5, \mathrm{p}=0.000$, Cramer's V=0.16)).

Interestingly, the usage of barrier protection methods in the form of wearing masks and gloves was associated with the same concerns as hand hygiene, but the relationship was inverse. The masks were worn by a minority of those who feared for the health of relatives and close people (Pearson's $\chi^{2}=47.9, p=0.000$; Cramer's $\mathrm{V}=0.16$ ), worried about lack of specific treatment for COVID-19 (Pearson's $\chi^{2}=32.9, p=0.000$, Cramer's V $=0.13$ ) and for their self-lives (Pearson's $\chi^{2}=29.0, p=0.000$, Cramer's $\mathrm{V}=0.12$ ). Also a minority used masks and gloves among respondents who feared for virus transmissibility (Pearson's $\chi^{2}=38.0, p=0.000$, Cramer's V $=0.14$; Pearson's $\chi^{2}=20.7, p=0.000$, Cramer's V $=0.10$ ) and those concerned with a lack of commercially available protection remedies (Pearson's $\chi^{2}=43.8, \mathrm{p}=0.000$, Cramer's V $=0.15$; Pearson's $\chi^{2}=22.6, p=0.000$, Cramer's V=0.11).

\section{Specifics of respondents' reactions to the pandemic}

On average, respondents reported 5 anxious concerns about coronavirus (Me; q25=4, q75=6) and 4 measures to 
prevent coronavirus infection (Me; $\mathrm{q} 25=3, \mathrm{q} 75=4)$ at the same time. Psychological stress indicators were significantly increased when only a few types of infection prevention measures were used (self-isolation, social distancing, and sanitizer usage by participants (Table 3 ).

It is important to note that subgroups of patients who showed significant differences in the severity of stress also had specific behavioral reactions to the pandemic. Thus, subgroups of young and middle-aged patients were more likely to use stress-related protective measures (self-isolation - individuals aged 18-40 (Pearson's $\chi^{2}=53.3, p=0.000, n=5$, Cramer's V=0). 17, effect average), principles of social distancing - respondents 2150 years old (Pearson's $\chi^{2}=18.6, p=0.002, n=5$, Cramer's $\mathrm{V}=0.1$, effect average), sanitizer usage - participants 21 30 years old (Pearson's $\chi^{2}=16.0, p=0.007, n=5$ Cramer's $\mathrm{V}=0.09$, effect average)). These age groups were more likely to resort to hand washing (Pearson's $\chi^{2}=25.0$, $\mathrm{p}=0.000, \mathrm{n}=5$, Cramer's $\mathrm{V}=0.11$, effect averaged). Masks were worn more often by respondents $31-60$ years old (Pearson's $\chi^{2}=23.6, \mathrm{p}=0.000, \mathrm{n}=5$ Cramer's $\mathrm{V}=0.11$, effect average) and gloves by respondents $41-78$ years old (Pearson's $\chi^{2}=35.0, \mathrm{p}=0.000, \mathrm{n}=5$ Cramer's $\mathrm{V}=0.13$, effect average).

Study participants from different health groups did not show significant changes in behavioral reactions. Thus, on the level of statistical but not practical significance, individuals with chronic diseases were more likely to neglect stress-related use of sanitizer than physically healthy respondents (Pearson's $\chi^{2}=8.1, p=0.004$, Cramer's V=0.06 - no effect). In addition, affective and somatic disorders were associated with fewer cases of mask wearing (Pearson's $\chi^{2}=8.2, \mathrm{p}=0.004$, Cramer's $\mathrm{V}=0.07$ - no effect; Pearson's $\chi^{2}=10.2, \mathrm{p}=0.001$, Cramer's $\mathrm{V}=0.07$ - no effect). Individuals with affective disorders were less likely to use gloves than study participants who did not report any mental disorders (Pearson's $\chi^{2}=10.8$, $\mathrm{p}=0.001$, Cramer's V $=0.07$ - no effect).

Table 2. Certain pandemic concerns in association with protective measures used by respondents ( $\%$ of the sample)

\begin{tabular}{|c|c|c|c|c|c|c|c|c|c|c|c|c|c|}
\hline & & \multicolumn{2}{|c|}{$\begin{array}{l}\text { Washing } \\
\text { hands }\end{array}$} & \multicolumn{2}{|c|}{$\begin{array}{l}\text { Self- } \\
\text { isolation }\end{array}$} & \multicolumn{2}{|c|}{$\begin{array}{l}\text { Physical } \\
\text { distancing }\end{array}$} & \multicolumn{2}{|c|}{$\begin{array}{c}\text { Use of } \\
\text { antiseptics }\end{array}$} & \multicolumn{2}{|c|}{$\begin{array}{l}\text { Wearing } \\
\text { masks }\end{array}$} & \multicolumn{2}{|c|}{$\begin{array}{l}\text { Wearing } \\
\text { gloves }\end{array}$} \\
\hline & & + & - & + & - & + & - & + & - & + & - & + & - \\
\hline \multirow{2}{*}{$\begin{array}{l}\text { Risk to the life and } \\
\text { health of relatives }\end{array}$} & + & 73.7” & 4.3 & 60.3” & 17.7 & $60.0 "$ & 18.0 & 49.0” & 29.0 & 33.1 & 44.8 & & \\
\hline & - & 18.5 & 3.5 & 14.4 & 7.6 & 13.6 & 8.4 & 8.9 & 13.1 & 5.3 & 16.7 & & \\
\hline \multirow{2}{*}{$\begin{array}{l}\text { Absence of specific } \\
\text { treatment for COVID-19 }\end{array}$} & + & $38.9^{*}$ & 1.4 & & & & & $26.3^{*}$ & 14.0 & 18.6 & 21.7 & & \\
\hline & - & 53.3 & 6.4 & & & & & 31.6 & 28.1 & 19.9 & 39.8 & & \\
\hline \multirow[t]{2}{*}{ Contagiousness of the virus } & + & $34.9^{*}$ & 1.2 & & & $29.2^{*}$ & 6.9 & $24.0 *$ & 12.2 & 17.2 & 19.0 & 9.5 & 26.7 \\
\hline & - & 57.3 & 6.6 & & & 44.4 & 19.5 & 33.9 & 30.0 & 21.3 & 42.5 & 11.2 & 52.6 \\
\hline \multirow[t]{2}{*}{ Fear for own life } & + & $30.5^{*}$ & 1.1 & & & & & $21.1^{*}$ & 10.5 & 14.9 & 16.7 & & \\
\hline & - & 61.6 & 6.7 & & & & & 36.7 & 31.7 & 23.5 & 44.8 & & \\
\hline \multirow{2}{*}{$\begin{array}{l}\text { Lack of protection } \\
\text { equipment for sale }\end{array}$} & + & $26.9^{*}$ & 0.9 & $22.7^{*}$ & 5.1 & & & & & 13.9 & 13.8 & 7.7 & 20.1 \\
\hline & - & 65.3 & 6.9 & 52.0 & 20.2 & & & & & 24.5 & 47.7 & 13.0 & 59.2 \\
\hline \multirow{2}{*}{$\begin{array}{l}\text { Possible lack of medication } \\
\text { for daily intake }\end{array}$} & + & & & & & $18.3^{*}$ & 3.9 & & & & & & \\
\hline & - & & & & & 55.3 & 22.5 & & & & & & \\
\hline
\end{tabular}

Table 3. Infection control measures in the sample (\%) and the level of associated psychological stress (PSM-25)

\begin{tabular}{|c|c|c|c|c|c|c|c|c|}
\hline \multirow{2}{*}{\multicolumn{2}{|c|}{$\begin{array}{l}\text { Practiced protection } \\
\text { measures }\end{array}$}} & \multicolumn{6}{|c|}{ Frequency of use among participants of different age groups } & \multirow{2}{*}{$\begin{array}{c}\text { PSM-25 } \\
(\mathrm{M} \pm \text { S.D. / Cohen's d) }\end{array}$} \\
\hline & & $18-20$ & $21-30$ & $31-40$ & $41-50$ & $51-60$ & $61-78$ & \\
\hline \multirow[t]{2}{*}{ Washing hands } & + & 14.7 & 41.5 & 16.6 & 10.8 & 6.2 & 2.4 & - \\
\hline & - & 1.1 & 2.4 & 1.9 & 1.0 & 0.7 & 1.6 & - \\
\hline \multirow[t]{2}{*}{ Self isolation } & + & 13.4 & 34.2 & 13.2 & 7.2 & 4.5 & 2.3 & $106.0 \pm 34.4 /<0.1$ \\
\hline & - & 2.5 & 9.7 & 5.4 & 4.6 & 2.5 & 0.7 & $105.4 \pm 33.7$ \\
\hline \multirow[t]{2}{*}{ Physical distancing } & + & 11.4 & 34.2 & 13.4 & 8.1 & 4.6 & 1.8 & $106.1 \pm 34.0 / 0.14$ \\
\hline & - & 4.4 & 9.7 & 5.1 & 3.7 & 2.3 & 1.1 & $101.3 \pm 35.1$ \\
\hline \multirow[t]{2}{*}{ Use of antiseptics } & + & 9.0 & 26.8 & 10.8 & 7.2 & 3.7 & 1.2 & $106.4 \pm 34.0 / 0.11$ \\
\hline & - & 6.8 & 17.1 & 8.5 & 4.6 & 3.3 & 1.8 & $102.7 \pm 34.8$ \\
\hline \multirow[t]{2}{*}{ Wearing masks } & + & 5.9 & 15.0 & 7.3 & 5.8 & 3.4 & 1.2 & - \\
\hline & - & 9.9 & 28.9 & 11.3 & 6.0 & 3.6 & 1.8 & - \\
\hline \multirow[t]{2}{*}{ Wearing gloves } & + & 2.2 & 8.0 & 4.4 & 3.1 & 2.4 & 0.6 & - \\
\hline & - & 13.6 & 35.9 & 14.1 & 8.7 & 4.5 & 2.4 & - \\
\hline
\end{tabular}




\section{DISCUSSION}

As a result of our study, we obtained data on specific associations of age, clinical and psychological factors with patterns of population behavior at the initial stages of restrictive measures due to the COVID-19 pandemic. The concerns about the availability of remedies on the free market were specifically related to the self-isolation strategies, and the combination of concerns about the infectiousness of the virus and the inaccessibility of drugs for daily use was associated with the use of physical distancing. Moreover, the concerns about the lack of specific treatment for COVID19 , the danger for self-lives, the infectiousness of the virus and the lack of protective equipment were associated with the hand hygiene protective behavior. In cases of masks wearing the same anxious concerns as for patterns of hand hygiene were prevalent, however their relationship was inverse. That could be due to conflicting information about the benefits of the mask regime presented in the media during the survey period. The most common type of concern (fear for loved ones) was not associated with a specific type of behavior, but rather with its specificity in terms of stricter adherence to the anti-epidemic regime.

Simultaneously, an increased level of psychological stress was associated with the physical distancing and the use of sanitizer. The distancing and perceptions of isolation were reported as potential initial factors of mental health symptoms in previous reports (Deng et al. 2021). Moreover, at the period of the survey, there was a certain deficit of the sanitizer in Russia. Thus, anti-epidemic measures (e.g. distancing and the necessity of sanitizer use) in the studied sample could be additional independent stress factors. This is consistent with literature data that even people who have not personally contacted the infection can experience the critical consequences of social restrictions (Lei et al. 2020). It is important to note here that the subjectivity of the population's perception of the recommended anti-epidemic measures can determine both the constructive nature of their response (social distancing) and destructive (avoidance of social contacts), which is associated with the options for the adaptive health anxiety (Asmundson \& Taylor 2020).

In the available to us scientific resources, there is extremely limited information on the association of psychological reactions of the population with the practiced protection measures at the initial stages of deterioration of the epidemiological situation and the announcement of quarantine restrictions (Rajkumar et al. 2020). We have received data indicating that at the initial stages of quarantine, some of the anxious concerns about COVID-19 are associated with the protective behavior of the population during a pandemic (self-isolation and the use of physical distancing). These include concerns about the availability of over- the-counter protective remedies, the infectiousness of the virus, and the unavailability of drugs for daily use. The problems of the certain medications unavailability and limitations on elective medical procedures due to pandemic were reported in previous studies and were very concerning, because of the difficulties they provided for the patients (Kaye 2020, Montemurro 2021). Moreover, there were reports that many patients with affective disorders had to discontinue their medication due to their unavailability in the epidemic period, which also resulted in increase in depression and anxiety (Gong et al. 2021).

The widespread use of hand hygiene among our respondents could be due to various factors. It is known that the Russian-speaking population is characterized by the primary use of those health care measures that are easier to implement in practice (Rasskazova et al. 2016). Previous studies have shown that simple hand-washing in the Chinese population was associated with a decrease in stress, anxiety and depression (Wang et al. 2020). It is important to note that the potential effectiveness of hand hygiene measures is most evident when the population does not adhere to self-isolation and distancing. This "secondary" nature of this measure is emphasized by its common psychological prerequisites with irrational behavior identified in the studied sample. Those are wearing masks and gloves by respondents who do not report concerns about the shortage of personal protective equipment and the infectiousness of the coronavirus, as well as the use of masks without expressing anxiety for self-life and inaccessibility of specific treatment for COVID-19. During the H1N1 and SARS epidemics, a similar example of the irrational behavior of the population of the United States and Hong Kong was an explosive increase in the number of people seeking medical care without sufficient reasons (Leung et al. 2005, McDonnell et al. 2012).

Of particular note is that different groups of the population, depending on their age, the history of somatic or affective pathology, showed different severity of anxiety distress associated with the pandemic. Furthermore, the diversity of psychological stress levels presented in age subgroups was associated with significant changes in behavioral patterns to prevent infection. Our results are in line with previous finding, which showed that the quarantine period led to the symptoms of anxiety, depression, worry and anger with variable behavior responses and emotional reactions due to isolation (Deng et al. 2021). Other studies also confirm the higher level of negative stress responses in vulnerable subgroups, such as adolescents and mentally ill individuals (Cloffi 2020, Joseph et al. 2020). According to our previous research among respondents with mood disorders and somatic burden high levels of psychological stress did not lead to adaptive behavior change (Sorokin et al. 2020). 


\section{Limitations}

The study had several potentially important limitations: 1) The obtained data were completely based on the results of self-report, including data on the history of mental and somatic pathology, which makes the objective assessment difficult. 2) The data were obtained for a sample in which women predominated significantly, which limits the direct extrapolation of the results to the entire Russian-speaking population. 3) When stratifying the sample by health groups, there was a shift in age in certain subgroups, which could also affect the results. 4) The patterns of associations between the behavior of the population, the severity of stress and the structure of anxious concerns that we obtained belong to the "search stage" of the study of the population's reactions to a pandemic and cannot be interpreted as causal. Nevertheless, in our opinion, they allow us to draw conclusions about the factors that are most closely related to the patterns of behavioral strategies of the population in the early stages of the COVID-19 pandemic.

\section{CONCLUSION}

The initial quarantine measures is an important stage that determines the containment of the epidemiological process. The increased level of psychological stress and a large variability of concerns associated with the pandemic are the result of an unprecedented situation in the spread of coronavirus infection and its widespread coverage in the media. At the same time, the connection between emotional experiences and behavioral reactions requires detailed study, since subjective perception of the recommended anti-epidemiological measures by the population is associated with a number of factors, which include age, the availability of correct information in the media, and the history of somatic and mental disorders. It is at the initial stage of the spread of COVID-19 that training in constructive ways to respond to a negative emotional background becomes especially important. The learning of constructive ways to overcome increased anxiety and distress by informing the population about behavioral strategies for overcoming it acquires a special role. Emphasis should be on particularly vulnerable groups of the population. This determines the need for early involvement of mental health professionals in the provision of care to the population and the providing focused recommendations.

\section{Acknowledgements:}

Non-governmental organization "Russian Society of Psychiatrists", the Interregional Professional Union "Physicians' Alliance", the educational portal "Psychiatry \& Neurosciences" and the information portal "Fontanka".

Conflict of interest: None to declare.

\section{Contribution of individual authors:}

Mikhail Sorokin proposed the idea of the study, developed the protocol for research, analyzed data, interpreted results, wrote the initial draft of the manuscript.

Evgeny Kasyanov proposed the idea of the study, developed the protocol for research, developed the online questionnaire, identified societies and social media groups to target participants, supervised data collection and data entry, interpreted results, wrote the initial draft of the manuscript.

Grigory Rukavishnikov developed the protocol for research, interpreted results, wrote the initial draft of the manuscript.

Olga Makarevich interpreted results, wrote the initial draft of the manuscript.

Nikolay Neznanov identified societies and social media groups to target participants, reviewed and edited the manuscript.

Natalia Lutova developed the protocol for research, identified societies and social media groups to target participants, reviewed and edited the manuscript.

Galina Mazo developed the protocol for research, identified societies and social media groups to target participants, reviewed and edited the manuscript.

\section{References}

1. Asmundson GJG \& Taylor S: How health anxiety influences responses to viral outbreaks like COVID-19: what all decision-makers, health authorities, and health care professionals need to know. J Anxiety Disord April 2020:

71:102211. https://doi.org/10.1016/j.janxdis.2020.102211

2. Brooks SK, Webster RK, Smith LE, Woodland L, Wessely $S$, Greenberg $N$ et al.: The psychological impact of quarantine and how to reduce it: rapid review of the evidence. Lancet March 14, 2020: 395:912-920. https://doi.org/10.1016/S0140-6736(20)30460-8

3. COVID-19 Dashboard by the Center for Systems Science and Engineering (CSSE) at Johns Hopkins University (JHU). September 2020.

https://gisanddata.maps.arcgis.com/apps/opsdashboard/in dex.html\#/bda7594740fd40299423467b48e9ecf6

4. Cioffi A: COVID-19, Mentally Ill and Mental Health Workers. Psychiatr Danub Summer 2020: 32:307.

5. Deng Y, Wang L, Yang J, Xie L, Chen Y: How COVID-19 Patient Narratives Concerning Reinfection Mirror Their Mental Health: A Case Series. Psychiatria Danubina Spring 2021: 33:114-119. https://doi.org/10.24869/psyd.2021.114

6. Duan L\& Zhu G: Psychological interventions for people affected by the COVID-19 epidemic. Lancet Psychiatry 2020; 7:300-302. https://doi.org/10.1016/S22150366(20)30073-0

7. Gong $H, D u X, W u L$ et al.: Discontinuation Syndrome of Extended-Release Venlafaxine during the COVID-19 Epidemic. Psychiatr Danub 2021; 33:121-122 
8. Huang Y \& Zhao N: Generalized anxiety disorder, depressive symptoms and sleep quality during COVID-19 outbreak in China: a web-based cross-sectional survey. Psychiatry Res June 2020: 288:112954. https://doi.org/10.1016/j.psychres.2020.112954

9. Joseph SJ, Bhandari SS, Ranjitkar S, Dutta S: School Closures and Mental Health Concerns for Children and Adolescents during the COVID-19 Pandemic. Psychiatr Danub Summer 2020: 32:309-310.

10. Kaye K, Paprottka F, Escudero $R$ et al.: Elective, Nonurgent Procedures and Aesthetic Surgery in the Wake of SARS-COVID-19: Considerations Regarding Safety, Feasibility and Impact on Clinical Management. Aesthetic Plast Surg 2020: 44(3):1014-1042. https://doi.org/10.1007/s00266-020-01752-9

11. Lei L, Huang X, Zhang S, Yang J, Yang L, Xu M: Comparison of Prevalence and Associated Factors of Anxiety and Depression Among People Affected by versus People Unaffected by Quarantine During the COVID-19 Epidemic in Southwestern China. Med Sci Monit April 26, 2020 Apr: 26:e924609. https://doi.org/10.12659/MSM.924609

12. Leung GM, Ho LM, Chan SK, Ho SY, Bacon-Shone J, Choy RY et al.: Longitudinal assessment of community psychobehavioral responses during and after the 2003 outbreak of severe acute respiratory syndrome in Hong Kong. Clin Infect Dis. June 15, 2005: 40:1713-1720. https://doi.org/10.1086/429923

13. Li Z, Ge J, Yang M, Feng J, Qiao M, Jiang $R$ et al.: Vicarious traumatization in the general public, members, and non-members of medical teams aiding in COVID-19 control. Brain Behav Immun August 2020: 88:916-919. https://doi.org/10.1016/j.bbi.2020.03.007

14. McDonnell WM, Nelson DS, Schunk JE: Should we fear "flu fear" itself? Effects of H1N1 influenza fear on ED use. Am J Emerg Med February 2012: 30:275-282. https://doi.org/10.1016/j.ajem.2010.11.027

15. Montemurro N: Intracranial hemorrhage and COVID-19, but please do not forget "old diseases" and elective surgery. Brain Behav Immun. 2021: 92:207-208. https://doi.org/10.1016/j.bbi.2020.11.034
16. Moore RC, Lee A, Hancock JT, Halley M, Linos E: Experience with Social Distancing Early in the COVID-19 Pandemic in the United States: Implications for Public Health Messaging. medRxiv [Preprint] April 2020: 11:2020.04.08.200570677. https://doi.org/10.1101/2020.04.08.20057067

17. Ozamiz-Etxebarria N, Dosil-Santamaria M, PicazaGorrochategui M, Idoiaga-Mondragon N: Stress, anxiety, and depression levels in the initial stage of the COVID-19 outbreak in a population sample in the northern Spain. Cad Saude Publica April 30, 2020: 36:e00054020. https://doi.org/10.1590/0102-311X00054020

18. Rajkumar RP: COVID-19 and mental health: A review of the existing literature. Asian J Psychiatr August 2020: 52:102066. https://doi.org/10.1016/j.ajp.2020.102066

19. Rasskazova EI \& Ivanova TU: Motivational models of health-related behavior: the problem of the "gap" between intention and action. Psych J Higher School Economics 2015: 12:105-130. (In Russ.)

20. Rasskazova EI, Tkhostov AS, Guldan VV: Ways of Taking Care of Health and Somatic Complaints in Normal: Role of Perception and Role of Action. Psych Res 2016; 9:4-13. (In Russ.)

21. Singh $R \&$ Adhikari R: Age-structured impact of social distancing on the COVID-19 epidemic in India. arXiv March 2020: arXiv2003.12055v.

22. Sorokin MY, Kasyanov ED, Rukavishnikov GV, Makarevich OV, Neznanov NG, Lutova NB et al.: Structure of anxiety associated with COVID-19 pandemic: the online survey results. Bull RSMU 2020; 3:77-84. https://doi.org/10.24075/brsmu.2020.030

23. Wang C, Pan R, Wan X, Tan Y, Xu L, Ho CS et al.: Immediate psychological responses and associated factors during initial stage of the 2019 Coronavirus Disease (COVID-19) epidemic among general population in China. Int J Environ Res Public Health March 6, 2020: 17:1729. https://doi.org/10.3390/ijerph17051729

Correspondence:

Evgeny D. Kasyanov, MD

V.M. Bekhterev National Medical Research Center for Psychiatry and Neurology 192019, Saint Petersburg, 3 Bekhterev St., Russian Federation

E-mail: ohkasyan@yandex.ru 